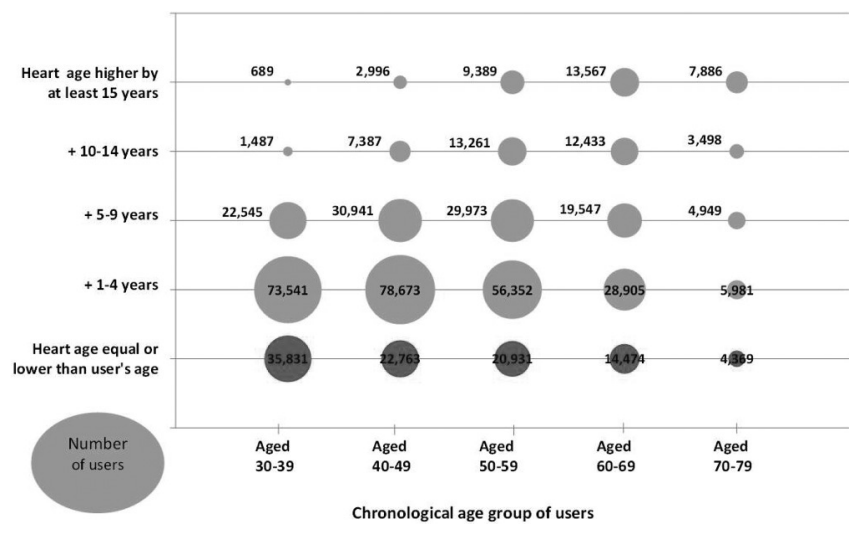

Abstract 83 Figure 2 Heart age estimates with proportions who are older then their heart age, by age group of users

of users lack awareness of their own risk factors. The Heart Age tool was accessed by a group not easily reached by conventional approaches yet is at high cardiovascular risk and would benefit most from early and sustained risk reduction. These are both important opportunities for interventions to educate and empower the public to manage better their cardiovascular risk and promote population level prevention.

\section{CARDIOVASCULAR RISK REDUCTION USING CONTEMPORARY GUIDELINE RECOMMENDATIONS: OUTCOMES OF THE HEART ATTACK PREVENTION PROGRAMME FOR YOU (HAPPY) LONDON STUDY}

Mohammed Khanji*, Armida Balawon, Redha Boubertakh, Steffen Petersen. Centre for Advanced Cardiovascular Imaging and Research, Queen Mary University London; *Presenting Author

\subsection{6/heartjnl-2016-309890.84}

Introduction Cardiovascular disease (CVD) remains the leading cause of morbidity and mortality globally. The importance of primary prevention (PP) of CVD is now well recognised and can potentially improve quality of life and survival costeffectively.

Aim To assess benefits of contemporary guideline based PP recommendations for CVD risk reduction in those with elevated global cardiovascular risk.

Methods 402 participants aged 40-74 years were recruited as part of the HAPPY London study. Eligible participants had an elevated 10-year risk based on a QRISK2 score of $10 \%$ or more. Internet access was required to be able to register and complete questionnaires online. Individualised one-off cardiovascular risk reduction advice was provided, based on the JBS3 and ESC 2012 CVD prevention guidelines. Follow-up at 3 months assessed changes in risk factors and risk scores using paired t-test statistical analysis.

Results Mean age 65 years; 37\% females and 370 (92\%) completed the 3-months follow up. There was a small but significant improvement in mean blood pressure from 132/80 $\mathrm{mmHg}$ to $129 / 78 \mathrm{mmHg}(\mathrm{p}<0.001)$. Other improvements included weight, waist circumference, fasting glucose, total cholesterol, physical activity, Framingham and QRISK2 scores (Table 1).

QRISK2 score dropped from $18.9 \%$ to $18.5 \%$ at followup. This was a $0.7 \%$ absolute reduction compared to expected
Abstract 84 Table 1 Baseline and follow up measurements

\begin{tabular}{llllll}
\hline Variable (mean) & Baseline & 3 Months & P value & $\mathbf{9 5 \%}$ & $\mathbf{C l}$ of differences \\
\hline Systolic BP (mmHg) & 132.4 & 129 & $<0.001$ & 2.36 & 4.77 \\
Diastolic BP (mmHg) & 79.6 & 78 & $<0.001$ & 1.00 & 2.35 \\
Weight (kg) & 80.3 & 79.2 & $<0.001$ & 0.55 & 1.02 \\
BMI (kg/cm2) & 27.7 & 27.4 & $<0.001$ & 0.18 & 0.34 \\
Waist circumference (cm) & 95.6 & 93.8 & $<0.001$ & 0.96 & 1.92 \\
Framingham score (\%) & 17.4 & 15.8 & $<0.001$ & 1.04 & 2.1 \\
QRISK2 score (\%) & 18.9 & 18.5 & 0.016 & 0.06 & 0.57 \\
Total cholesterol & 5.0 & 4.8 & $<0.001$ & 0.17 & 0.31 \\
Glucose & 5.8 & 5.6 & $<0.001$ & 0.1 & 0.3 \\
LDL & 2.8 & 2.7 & $<0.001$ & 0.09 & 0.22 \\
\hline
\end{tabular}

resulting in about 660 less CVD events per 100,000 over 10 years.

Conclusions Implementing guideline recommendation for CVD PP can produce modest improvements in risk factors in the medium term in patients at high cardiovascular risk. Promoting long-term compliance should help lower future cardiovascular events.

\section{SOFT DRINKS AND SWEETENED BEVERAGES AND THE RISK OF CARDIOVASCULAR DISEASE AND MORTALITY A SYSTEMATIC REVIEW AND META-ANALYSIS}

Aditya Narain. Keele University

\subsection{6/heartjnl-2016-309890.85}

Background Despite the well-characterised association between sweetened beverage intake and development of cardio-metabolic risk factors, the relationship between sweetened beverage intake and cardiovascular disease and mortality is controversial. A systematic review and meta-analysis was conducted to evaluate the relationship between sweetened beverages and cardiovascular events and mortality.

Methods Medline and EMBASE were searched in July 2015 for studies that considered soft drink intake and their association with risk of mortality, coronary heart disease (CHD) or stroke. Pooled risk ratios for adverse outcomes were calculated using inverse variance with a random effects model, and heterogeneity was assessed using the $\mathrm{I}^{2}$ statistic.

Results 8 studies with 308,810 participants (34-75 years) were included in the review. Pooled results suggest a significant increase in stroke RR 1.13 95\% CI 1.02-1.24, and CHD RR $1.2295 \%$ CI 1.14-1.30 with incremental increase in sugarsweetened beverage (SSB) consumption. For stroke and CHD, there were significant increases with greater incremental increase in artificially sweetened beverage (ASB) consumption (stroke RR 1.13 95\% CI 1.02-1.24, CHD RR 1.22 95\% CI 1.14-1.30), but not vascular events. For high versus low SSB, there was a significant increase in CHD (RR 1.19 95\% CI 1.09-1.31) but not stroke, vascular events or mortality. For ASB, there was a significant increase in stroke (RR 1.14 95\% CI 1.04-1.26) and vascular events (RR 1.44 95\% CI 1.02-2.03) but not CHD.

Conclusions Current evidence suggests an association between increased cardiovascular risk and greater consumption of sugar-sweetened beverages, although consumption may be a surrogate for adverse health behaviours. 